\title{
Inverse correlation between SMN1 and SMN2 copy numbers: evidence for gene conversion from SMN2 to SMN1
}

\author{
Shuji Ogino, Sizhen Gao, Debra GB Leonard, Michele Paessler and Robert B Wilson
}

European Journal of Human Genetics (2003) 11, 723. doi:10.1038/sj.ejhg.5201032

Addendum to: European Journal of Human Genetics (2003) 11, 275-277. doi:10.1038/sj.ejhg.5200957

In addition to our hypothesis of gene conversion from SMN2 to SMN1, our data are consistent with an alternative hypothesis for the presence of chromosome $5 \mathrm{~s}$ with two copies of SMN1. Rochette et al (Hum Genet 2001; 108: 255266) have provided evidence that the SMN1 gene duplicated before the separation of human and chimpanzee lineages, and that SMN2 appeared for the first time in Homo sapiens. The presence of chromosome 5s with two SMN1 copies could represent a remnant of the original duplication. If this were true, however, the much higher prevalence of chromosome $5 \mathrm{~s}$ with one copy each of SMN1 and SMN2 would suggest a selective advantage relative to chromosome $5 \mathrm{~s}$ with two copies of SMN1. Evidence for such a selective advantage is currently lacking. 